\title{
ABSTRACTS IN ECHOCARDIOGRAPHY 1999
}

\section{A survey of current practice of transoesophageal echocardiography in the UK: are recommended guidelines being followed?}

N Sutaria, D Northridge, M Denvir; Western General Hospital, Edinburgh

Transoesophageal echocardiography (TOE) has become established as a safe and effective imaging technique and is performed by cardiologists and sonographers using intravenous sedation. We investigated the variability in the practice of TOE in the UK and whether published guidelines on the administration of sedation by nonanaesthetists were being followed. Questionnaires were received from 45 centres ( 30 tertiary, 15 DGH) which included 31 consultants, 11 registrars and 3 technicians with TOE experience ranging from 20 to 2000 scans (median 300 ). $49 \%$ of operators routinely used sedation in all patients, $80 \%$ used sedation in most ( $>90 \%)$. Midazolam was used universally, mean dose $4 \mathrm{mg}$ (range $2-10$ ), in $71 \%$ this was by titration rather than bolus. $93 \%$ routinely used lignocaine throat spray. $100 \%$ of responders had immediate access to flumazenil, resuscitation equipment and suction. $60 \%$ had facilities for tilting head down. The procedure was performed with a second member of staff in $44 \%$ and with 2 other staff in $66 \% .64 \%$ attempted to maintain verbal communication in all cases and $58 \%$ reported patients frequently becoming verbally unresponsive. An oxygen supply was present in the procedure room in $93 \%$. Contrary to the published guidelines, routine administration of inspired oxygen and use of pulse oximetry was reported in only $20 \%$ and $31 \%$ of responses respectively. Furthermore, four operators $(8 \%)$ did not require intravenous access in all patients, and $47 \%$ rarely or never monitored arterial blood pressure. $30 \%$ of responders used prophylactic antibiotics in selected cases. $74 \%$ of operators had not received training in the administration of sedation and only $31 \%$ were aware of any guidelines.

Conclusions: There is wide variation in the practice of TOE in the UK. Most people regularly performing TOEs with intravenous sedation have not been trained in the administration of sedation and do not adhere to national guidelines, particularly with respect to the routine use of oxygen and oximetry.

Tissue harmonic imaging improves wall motion analysis during dobutamine stress echo

T Zaglavara, M Norton, B Cumberledge, D Morris, T Irvine, C Cummins, J Schuster, A Kenny; Department of Cardiology, Freeman Hospital, Newcastle upon Tyne

We performed a study to determine whether tissue harmonic imaging (THI) facilitates wall motion analysis at rest and whether these benefits extend through the stages of a dobutamine stress echocardiography (DSE) study. We also assessed the impact of THI on the feasibility of DSE in technically difficult patients.

Twenty unselected patients underwent DSE by standard protocol. Parasternal and apical views were obtained using fundamental mode (FND) and THI, at baseline, low dose and peak stress. Segmental endocardial border definition (EBD) was characterised as $1-4(1=$ excellent) and segmental wall motion was characterised as $1-4 / x(1=$ normal, $\mathrm{x}=$ unable to interpret) by a consensus of 2 experienced observers.

EBD improved with THI in $26 \pm 6.7$ of the 48 segments per patient $(54 \%)$ and deteriorated with THI in only $2 \pm 2.7(4 \%)$. Of the 48 segments per patient a mean of $10 \pm 5.7(21 \%)$ were of inadequate quality to be interpreted for wall motion on FND, and this changed to $4 \pm$ $3.4(6 \%)$ on THI $(p<0.001)$. EBD improved in a similar degree in all DSE stages $53 \%, 54 \%$ and $53 \%$ for rest, low dose and peak stress respectively. Six of the $20(30 \%)$ study patients were deemed unsuitable for DSE on FND ( 5 or more of the 16 segments inadequately visualised on resting echo). In particular, a mean of $6 \pm 0.75$ segments per patient were inadequately visualised on resting echo using FND, and this changed to $2 \pm 1.6$ on THI. Thus, all subjects changed to suitable for DSE using THI $(\mathrm{p}<0.01)$. Of the total 205 segments deemed unsuitable for interpretation on FND 140 (68\%) comprised the anterior and lateral walls of the LV. Improvement with THI was also more prominent on these walls.
THI dramatically improves EBD and the ability to confidently score segmental wall motion. These benefits extend to the peak stage of a DSE study. Routine use of THI may enhance the diagnostic accuracy of DSE and extend its application to technically difficult patients previously deemed unsuitable.

Regional improvement in left ventricular function following acute myocardial infarction can be predicted using ultrasonic integrated backscatter

J Hancock, D Chin, D E Jewitt, M J Monaghan; King's College Hospital, London

Cyclic variation in ultrasonic integrated backscatter (cyclic IBS) is reduced in myocardial segments supplied by the infarct related artery (IRA) following myocardial infarction (MI), probably due to a reduction in microvascular pulsatile flow. Cyclic IBS should return to normal levels if patency of the IRA can be restored, either with thrombolysis or by coronary angioplasty (PTCA), provided that microvascular perfusion is re-established. Thirty patients who had sustained an acute MI were studied. Coronary angiography was performed following thrombolysis to determine patency of the IRA. If this was occluded, PTCA was performed to restore TIMI 3 flow. This was achieved in 28/30 patients. Cyclic IBS was measured in myocardial segments supplied by the IRA pre thrombolysis (where possible), post thrombolysis, pre discharge and at 1 month and 3 months. Additionally, a wall motion score index (WMSI) was determined by dividing the total wall motion score by the number of segments scored using a scoring system of $1=$ normal, $2=$ hypokinetic, $3=$ akinetic, 4 $=$ dyskinetic. An increase in cyclic IBS of $>30 \%$ predischarge compared with the initial value was used to predict recovery of wall motion. 12 patients had almost no change in their cyclic IBS pre discharge $(0.18 \mathrm{~dB}, 6 \%)$ whereas 18 had a significant increase $(2.4 \mathrm{~dB}$, $38 \% ; \mathrm{p}<0.0001)$. All patients had similar WMSI pre discharge $(1.9$, NS). Patients whose cyclic IBS had increased significantly pre discharge had a marked improvement in their WMSI at 1 and 3 months $(1.17 ; \mathrm{p}<0.0001)$. In conclusion, cyclic IBS can be used to predict which patients will have recovery of myocardial function following MI.

LV systolic function post MI-a comparison of three quick methods

G A Cooke, G McGawley, J Wolfenden, K E Berkin; Cardiology Department, St James's Teaching Hospital, Leeds

The prognosis after myocardial infarction (MI) is closely related to LV systolic function, and can be improved by ACE inhibitors. Therefore requests to assess LV systolic function are numerous, and the assessments can sometimes be difficult, inaccurate and time-consuming. In many instances a subjective assessment is reported with no measure of ejection fraction (EF). The TRACE study showed that a simple 9 segment scoring system was accurate enough to stratify post-MI patients according to prognosis. Recently longitudinal contraction (MV displacement, MVD) has been proposed as simple, quick and of prognostic significance.

We compared the subjective (S), TRACE (T) and MVD methods in 67 patients $1-5$ days post MI. The S EF was reported in $10 \%$ bands (from less than $20 \%$ to $50 \%$ or more) immediately to the clinician on CCU. The T and MVD EF were calculated later without reference to the S EF. The T EF method involved scoring 4 basal, 4 mid and 1 apical segment (dyskinetic -1 , akinetic 0 , hypokinetic 1, normal 2). The total score was divided by the number of segments scored and multiplied by $30 \%$ to obtain T EF. The MVD was measured from the apical views by $\mathrm{M}$-mode placed on the medial, lateral, anterior and inferior parts of the MV ring and a mean value calculated. The 3 methods were compared for equivalence and ease.

There was excellent agreement between the S and T EF with only 1 minor discrepancy, which did not alter the decision to prescribe an 
ACE inhibitor. The MVD EF showed no correlation with the other 2 methods. The $\mathrm{S} E \mathrm{EF}$ was quicker than the T EF, which added 1-2 mins to reporting time. The MVD method required additional recording and reporting time (3-4 mins).

The $\mathrm{S}$ and $\mathrm{T}$ EF methods are quick, simple and show good agreement, suggesting accuracy and prognostic usefulness. The MVD method appears less useful post MI than reported in chronic heart failure.

\section{Quantitation of pulmonary regurgitation and right ventricular function using a 4D digital colour Doppler reconstruction method and real time 3D colour Doppler imaging: an in vivo study in a chronic animal model}

RA Ruski, ${ }^{1}$ X-N Li, ${ }^{2}$ T Irvine, ${ }^{1}$ Y Mori,,${ }^{1}$ M Jones, ${ }^{3}$ J A Panza, ${ }^{3}$ V Sachdev, ${ }^{3}$ D Lennon, ${ }^{2}$ A Kenny, ${ }^{4}$ DJ Sahn

${ }^{1}$ Oregon Health Sciences University, Oregon, USA $;{ }^{2}$ ATL Ultrasound, Bothell, Washington, USA; ${ }^{3}$ NHLBI, Bethesda, Maryland, USA; ${ }^{4}$ Freeman Hospital, Newcastle upon Tyne

Accurate non invasive quantitation of pulmonary regurgitation and right ventricular (RV) function are important in patients with congenital heart disease or pulmonary hypertension. In 4 open chested sheep, scanned epicardially 6-8 weeks after pulmonary valvectomy, we applied two $3 \mathrm{D}$ echocardiographic techniques to assess pulmonary regurgitation and RV function. Recordings from electromagnetic flowmeters placed on the aorta and pulmonary artery were used as reference. For the first method, ECG gated digital 3D velocity data were acquired for forward and reverse flow across the RVOT in 14 haemodynamic states. An ATL HDI 5000 scanner was used and off-line flow quantitation was performed on a Unix based computer by temporally integrating the digital velocities over a curved 3D surface perpendicular to the direction of interrogation. The 3D derived forward stroke volumes (SV) correlated well with EM data $(r$ $=0.86)$ as did pulmonary regurgitant volumes $(r=0.97)$ and regurgitant fractions $(r=0.95)$. The second method used real time grey scale and colour Doppler 3D echocardiography (Volumetrics scanner). Pulmonary forward and reverse flows were analysed as C scan cross sections just below the pulmonary valve. A ratio of the maximum flow area of reverse to forward flow correlated well with the EM regurgitant fractions $(r=0.82)$. The RV chamber volumes in diastole and systole were measured off line and SV computed. These also showed good correlation with EM SV data $(r=0.74)$. At present real time $3 \mathrm{D}$ lacks the sampling rate for $3 \mathrm{D}$ colour that is required for accurate flow quantitation and for which we used our new reconstruction method. In the future, real time 3D should provide cavity, stroke volume and flow quantitation.

\section{The On-X bileaflet replacement heart valve: initial} echocardiographic results

J Chambers, J Ely on behalf of the MCRI Investigators; Cardiothoracic Centre, Guy's and St Thomas' Hospital and MCRI.

Background: The On-X heart valve is a new bileaflet design with a shaped orifice and flared inlet to reduce inlet turbulence, an elongated orifice to reduce exit losses and thin leaflets able to align with flow to minimise obstruction. This is a report from the multicenter MCRI trial.

Methods: We studied 185 patients, 129 in the aortic and 72 in the mitral position at 10 centres in Europe between Sept 1996 and Oct 1998. The mean age was 60.5 years (range $20-80$ ). The echocardiography machines were calibrated using a phantom and every recording was checked by one observer. Data were collected according to American and British Society of Echocardiography and FDA guidelines and included effective orifice area $\left(\mathrm{EOA}\right.$ in $\left.\mathrm{cm}^{2}\right)$ by the continuity equation and peak and mean pressure drop $(\mathrm{dP}$ in $\mathrm{mm} \mathrm{Hg}$ ) using the Bernoulli theorem.

Results: For the aortic valves, mean $\mathrm{dP}$ ranged from $11.7 \mathrm{~mm} \mathrm{Hg}$ to $4.8 \mathrm{~mm} \mathrm{Hg}(\mathrm{p}<0.05)$ and EOA from $1.5 \mathrm{~cm}^{2}$ to $2.6 \mathrm{~cm}^{2}(\mathrm{p}<0.01)$ comparing the $19 \mathrm{~mm}$ and $27 / 29 \mathrm{~mm}$ valves. For valves in the mitral position, the mean $\mathrm{dP}$ was similar for all sizes and ranged from 4.7 to $5.0 \mathrm{~mm} \mathrm{Hg}$; the EOA ranged from 2.0 to $2.4 \mathrm{~cm}^{2}$. Minor pivotal washing jets were seen in 29 (48\%) aortic valves.

\begin{tabular}{lrrrrr}
\hline & $\begin{array}{l}19 \mathrm{~mm} \\
n=14\end{array}$ & $\begin{array}{l}21 \mathrm{~mm} \\
n=22\end{array}$ & $\begin{array}{l}23 \mathrm{~mm} \\
n=48\end{array}$ & $\begin{array}{l}25 \mathrm{~mm} \\
n=25\end{array}$ & \multicolumn{1}{c}{$\begin{array}{l}27 / 29 \mathrm{~mm} \\
n=16\end{array}$} \\
\hline Peak dP & $23.6(8.1)$ & $17.5(7.5)$ & $14.9(7.7)$ & $13.2(8.4)$ & $11.7(6.6)$ \\
Mean dP & $11.7(3.4)$ & $10.6(6.4)$ & $8.3(5.2)$ & $7.8(4.9)$ & $\begin{array}{r}4.8(2.1) \\
\text { EOA }\end{array}$ \\
\hline
\end{tabular}

Conclusion: The initial postoperative results show favourable haemodynamic performance for this new design of bileaflet valve.

\section{Left ventricular wall thickness assessment with tissue harmonic imaging}

M Norton, T Irvine, T Zaglavara, C Cummins, J Schuster, B Cumberledge, A Kenny; Cardiology Department, Freeman Hospital, Newcastle upon Tyne

Tissue harmonic imaging (THI) significantly improves endocardial visualisation. Many echocardiography departments use THI as the default setting for their cardiac ultrasound machines. We have become aware that THI can cause cardiac structures to appear thicker during harmonic imaging than during fundamental imaging. We therefore performed a systematic study to assess the effect of THI on echocardiographic measurements of left ventricular wall thickness. Twenty one consecutive patients, who were referred for transthoracic echocardiography, were studied. All patients were imaged using standard ATL HDI 5000 or HDI 3000 cardiac ultrasound systems. $\mathrm{M}$-mode measurements of interventricular and posterior left ventricular wall thickness were made at end diastole during both fundamental and tissue harmonic imaging.

The mean thickness of the interventricular septum was $1.09 \mathrm{~cm}$ when measured during fundamental imaging. This increased to 1.25 $\mathrm{cm}$ with THI. This $14.7 \%$ increase is statistically significant $(\mathrm{p}<$ 0.0001 , paired $t$ test). There was a similar increase in measurements of posterior left ventricular wall thickness from a mean of $1.02 \mathrm{~cm}$ in fundamental mode to $1.19 \mathrm{~cm}$ in THI mode. This $16.7 \%$ increase was also statistically significant $(\mathrm{p}<0.0005$, paired $t$ test). THI significantly increases the apparent thickness of both interventricular and posterior left ventricular walls. These results have important implications for echocardiographic assessment of the left ventricle. This study suggests that THI should not be used when measuring wall thickness.

Pulse inversion imaging with NC 100100 contrast agent: new techniques for improving left ventricular endocardial definition

J C Cooke, J E Hancock, D J Patsouras, D A Klotsa, M J Monaghan; King's College Hospital, London

Pulse inversion imaging (PI) is a new imaging technique designed to offer improved sensitivity for detection of ultrasound contrast agents. However, no human studies have been described to validate its theoretical benefits, especially in combination with 3rd generation ultrasound contrast agents. PI utilises 2 sequential ultrasound pulses, 180 degrees out of phase with each other, transmitted down each scan line. The returning pulse echoes are added together so that phase cancellation will occur when they are scattered from a linear reflector. Ultrasound contrast agents are non-linear and therefore incomplete cancellation occurs, resulting in a strong harmonic signal and high sensitivity for detection. We hypothesised that this technology should enhance our ability to detect the endocardial border. Twelve patients (8 male, aged 39-68) were imaged with PI using an ATL HDI 5000 during intravenous injection of NC 100100 (Nycomed Amersham), a new 3rd generation ultrasound contrast agent. Parasternal long-axis, short-axis, and apical 4 and 2 chamber views were recorded. Endocardial definition with and without contrast was analysed by 2 independent observers using a standard 16 segment model and a scoring system of $0=$ endocardium not visualised; $1=$ endocardium barely visible but sufficient; 2 = good endocardial definition. A total of 220 segments were analysed with and without contrast. There was a significant increase in the average endocardial definition score per segment following the use of contrast (1.309 compared to $1.918 ; \mathrm{p}<$ $0.0001)$. The number of interpretable segments also rose significantly from $83 \%$ to $100 \%$. In conclusion, the new imaging modalities of PI and NC 100100 provide good enhancement of the endocardial 
border and are likely to have an important role during difficult imaging situations such as stress echo.

\section{Limitations in the modified continuity equation}

J Chambers, J Ely; Guy's and St Thomas' Hospitals, London; and MCRI, Austin, Texas, USA

Background: The continuity equation is universally used for the estimation of orifice area for valves in the aortic position. The velocity ratio is usually thought to be equivalent to the more correct ratio of the velocity integral. This assumes that waveform shape above and below the valve is the same which may not be true.

Methods: We compared effective orifice area by the modified method using the velocity ratio (EOAmod) and the classical formula using the velocity integral (EOAclass) in 123 patient implanted with an On-X bileaflet mechanical valve. All were functioning normally in the immediate postoperative period. Valve sizes were $19 \mathrm{~mm}(\mathrm{n}=12), 21$ $\mathrm{mm}(\mathrm{n}=24), 23 \mathrm{~mm}(\mathrm{n}=45), 25 \mathrm{~mm}(\mathrm{n}=26)$ and $27 / 29 \mathrm{~mm}(\mathrm{n}=$ 16). Analysis was by simple regression, Bland-Altman analysis and by a Student's $t$ test.

Results: There was a good correlation between the two formulae $(r=$ $0.91)($ EOAmod $=0.9 \times$ EOAclass +0.1$)$. Despite this there were significant differences between the two methods using a Student's $t$ test $(\mathrm{p}<0.05)$. The results using the velocity ratio were a mean of $0.2 \mathrm{~cm}^{2}$ lower with $95 \%$ limits for the difference of -0.6 to $1.0 \mathrm{~cm}^{2}$.

\begin{tabular}{llll}
\hline & Geometric OA & EOAclass & EOAmod \\
\hline $19 \mathrm{~mm}$ & 2.0 & $1.5(0.2)$ & $1.2(0.3)$ \\
$21 \mathrm{~mm}$ & 2.5 & $1.8(0.4)$ & $1.5(0.5)$ \\
$23 \mathrm{~mm}$ & 3.1 & $2.3(1.0)$ & $2.0(0.6)$ \\
$25 \mathrm{~mm}$ & 3.7 & $2.6(1.0)$ & $2.4(0.6)$ \\
$27 / 29 \mathrm{~mm}$ & 3.7 & $2.8(0.5)$ & $2.6(0.6)$ \\
\hline
\end{tabular}

Conclusion: Previous comparisons using regression analysis may have disguised limitations of the modified continuity equation. The modified equation based on the velocity ratio cannot be substituted for the more correct velocity integral ratio in normally functioning bileaflet mechanical valves.

Detection of commissural calcification in patients undergoing balloon mitral valvotomy

N Sutaria, B Prendergast, T R D Shaw, D Northridge; Western General Hospital, Edinburgh

Aims: To investigate the incidence of commissural calcification, the role of transthoracic echocardiography (TTE) in its detection and the influence of commissural calcium on haemodynamic variables preand post-balloon mitral valvotomy (BMV).

Methods: Retrospective study of 345 patients, mean age 60 years, referred for BMV between 1986 and 1998. TTE was used to image the mitral valve in the short axis parasternal view and both anterolateral and posteromedial commissures were examined for the presence of calcium as evidenced by bright echo reflections. In a subset of 31 patients biplane TOE was used to image each commissure in the transverse and longitudinal mid-oesophageal planes and the accuracy of TTE was assessed using TOE as the "gold standard".

Results: $104(30 \%)$ patients had commissural calcification. TTE detected commissural calcification with a sensitivity of $92 \%$ and specificity of $81 \%$ (interobserver variation $8 \%$ ). TOE provided adequate visualisation in $100 \%$ of cases with an interobserver variation of $1.6 \%$. The incidence of commissural calcium increased significantly with age and was more common in males. These patients were more symptomatically limited, had more extensive leaflet and subvalvular deformity (mean Wilkins score $8.3 v 6.2, \mathrm{p}<0.001$ ) and had more severe mitral stenosis (mean pre-valve area $0.8 v 1.0 \mathrm{~cm}^{2}$, mean gradient $15 v 12 \mathrm{~mm} \mathrm{Hg}, \mathrm{p}<0.01)$. Commissural calcification was associated with a worse immediate haemodynamic outcome (mean post-valve area $1.5 v 1.8 \mathrm{~cm}^{2}$, mean gradient post-BMV $7 v 5 \mathrm{~mm} \mathrm{Hg}$, both $\mathrm{p}<0.001$ ).

Conclusions: Commissural calcification is common in the elderly and associated with a worse outcome from BMV. TTE is a sensitive technique for the detection of commissural calcium although in a significant minority of patients precordial images are inadequate and calcium may be incorrectly localised within either commissure; TOE is of additional value in these cases.

\section{Doppler derived exercise haemodynamics of the intact (Medtronic) aortic bioprosthesis at second review: evidence of favourable left ventricular remodelling}

B S Rana, D Gardiner, L Drummond, J L Low, S A Livesey, I A Simpson; Wessex Cardiothoracic Centre, Southampton University Hospitals

Exercise haemodynamic performance data of bioprosthetic valves in the aortic position is limited. There have been concerns that stented bioprosthetic valves may cause unacceptably high transprosthetic gradients at high flow rates. To examine this further we studied the rest and exercise haemodynamics of 67 patients who underwent aortic valve replacement with the Intact porcine valve, implanted between 1993 and 1996, at two time intervals. The implant sizes in the majority were $23 \mathrm{~mm}$ and $25 \mathrm{~mm}$ (range 21-29). The same patients were studied at each review using symptom limited treadmill exercise (mean of 21 months following implantation at first review, and 38 months at second review). Heart rate, blood pressure, cardiac output and left ventricular function were comparable between reviews. The echocardiographic Doppler derived peak and mean aortic valve gradients (AVG), and effective orifice area (EOA) and EOA index are expressed as mean (SD) as follows:

\begin{tabular}{|c|c|c|c|c|}
\hline & \multicolumn{2}{|l|}{ Rest } & \multicolumn{2}{|l|}{ Exercise } \\
\hline & Review 1 & Review 2 & Review 1 & Review 2 \\
\hline Peak AVG mmHg & $27(12)$ & $21.1(94)$ & $40.4(13.3)$ & $29(13)$ \\
\hline $\begin{array}{c}\text { Mean AVG } \\
\text { mmHg }\end{array}$ & $15(6.7)$ & $12.3(57)$ & $22.9(8.6)$ & $16.6(7.8)$ \\
\hline $\mathrm{EOA} \mathrm{cm}^{2}$ & $1.33(0.47)$ & $1.25(0.48)$ & $1.32(0.45)$ & $1.3(0$ 53) \\
\hline EOAI $\mathrm{cm}^{2} / \mathrm{m}^{2}$ & $0.73(0.25)$ & $0.68(0.24)$ & $0.73(0.26)$ & $0.71(0.27)$ \\
\hline $\mathrm{LVMI} \mathrm{g} / \mathrm{m}^{2}$ & $149.4(51.6)$ & $130.9(46.3)$ & & \\
\hline
\end{tabular}

By calculating the change in each of the variables from rest to exercise for each patient, a mean change was determined for the group. A significant fall in mean and peak AVG $(\mathrm{p}<0.005)$ at second review was found, while the EOA and EOAI did not change significantly ( $\mathrm{p}=$ 0.24 and 0.41 respectively). Echo derived left ventricular mass index (LVMI) was obtained in 41 patients and showed a significant fall over the same period $(p<0.005)$. In conclusion this stented bioprosthetic valve shows acceptable exercise haemodynamics which is supported by evidence of significant left ventricular regression.

A survey of the provision of echocardiography in South Thames

J Chambers, R Buckle; Guy's and St Thomas' Hospitals, South Thames Region, London

There are few data on the provision of echocardiography within the UK. Information is needed to aid the establishment of minimum standards for practice within the National Services Framework and local Health Improvement Plans. We therefore sent questionnaires to consultant cardiologists at all 30 hospitals in South Thames. The response rate was $18(60 \%)$ including 3 regional and 15 district hospitals (DGH). The regional hospitals had 557-1326 beds and 4.5-7.3 cardiologists. The DGH varied in size between 197 and 716 beds and there were $0.3-3$ consultant cardiologists. The number of echos performed each year was 4465-11 013 at the regional and 1010-2875 at the district hospitals. This was 20 to 113 studies indexed per 100 outpatients seen or between 1.0 and 5.4 per exercise test. Most studies were performed by technicians: $12-95 \%$ by an MTO5 in $11 \mathrm{DGH}$, $25-95 \%$ by an MTO4 at 12 , and $20-50 \%$ at 8 DGH by an MTO3. Clinicians performed or reported a median proportion $<5 \%$ of all studies. There were 18 technicians at the regional hospitals and 9 in training; while at the DGH there were 36 with 19 in training. Only 12 (67\%) regional and 15 (47\%) DGH technicians had BSE accreditation. Audit and business meetings occurred at all regional and only 2 (13\%) DGH, formal teaching at all regional and 5 (33\%) DGH. Paediatric studies were performed by all regional and $6(40 \%) \mathrm{DGH}$. TOE were performed at all regional and $6(40 \%)$ DGH and represented $3.6-4.8 \%$ of the total in regional and $0.7-3.1 \%$ in DGH. 
Stress echoes were performed at all regional and 3 DGH. Open access was offered by all regional and $10(67 \%)$ DGH.

Conclusion: Echocardiographic activity and organisation vary widely within South Thames. There appears to be inadequate clinical supervision, audit and continuing education. National planning of cardiac expansion within echocardiography including open access would require an initial investment to improve existing services.

\section{Right atrial pressure: comparison of echocardiography and} direct catheter measurement

D Mullins, E Tomsett, S Redwood, J Chambers; Guy's and St Thomas' Hospitals, London

Background: An estimate of right atrial (RA) pressure is required for the calculation of pulmonary artery pressure by Doppler. A number of empirical methods based on IVC size or venous flow patterns are in use but have not been fully validated.

Methods: We studied 17 patients, mean age 58 years (range 23-78 years) of whom 10 were male. Right atrial mean pressure was measured directly with a fluid-filled $6 \mathrm{~F}$ end-hole catheter at the end of routine coronary angiography or angioplasty. The transducer was calibrated before each reading. An echocardiogram was then performed within 5 minutes with the patient still lying flat and before any intervention likely to affect intracardiac pressures.

Results: RA mean pressures ranged from 4 to $17 \mathrm{~mm} \mathrm{Hg}$. These correlated with IVC diameter $(r=0.57 ; \mathrm{p}<0.05), \%$ IVC contraction during forced inspiration $(r=0.48 ; \mathrm{p}<0.05)$ and SVC systolic-atrial reversal $(r=0.52 ; \mathrm{p}<0.05)$. A model based on all three echo parameters correlated with $r=0.78(\mathrm{p}=0.006)$. There was no correlation between RA mean pressure and IVC cross-sectional area, \%IVC collapse with sniffing, hepatic atrial systolic-atrial reversal.

Conclusion: Easily obtained echocardiographic parameters correlate with invasively measured right atrial pressure and may prove to be clinically useful.

\section{Left atrial appendage visualisation using tissue harmonic imaging}

M Norton, J Schuster, T Zaglavara, B Cumberledge, C Cummins, C Plummer, J Indrakumar, A Kenny; Cardiology Department, Freeman Hospital, Newcastle upon Tyne

Left atrial appendage (LAA) thrombus is an important risk factor for embolic stroke. Transoesophageal echocardiography (TOE) is the gold standard for LAA assessment. Tissue harmonic imaging (THI) improves left ventricular endocardial definition during transthoracic echocardiography (TTE). We hypothesised that THI would increase our ability to visualise the LAA using TTE. 50 consecutive patients referred for TTE were scanned using a conventional cardiac ultrasound system. The LAA was examined in both fundamental (FND) and THI modes. Images were reviewed offline by two independent observers. The LAA was divided into four segments and endocardial definition was scored as follows: $0=$ not visible, $1=$ inadequately seen, 2 = just adequately visualised, 3 = good visualisation, 4 $=\mathrm{TOE}$ quality. THI significantly increased the visualisation scores.

Endocardial visualisation scores

\begin{tabular}{llll}
\hline Segment & FND score & THI score & p Value \\
\hline 1 & 1.93 & 2.20 & $<0.05$ \\
2 & 1.33 & 1.59 & $<0.05$ \\
3 & 1.42 & 1.72 & $<0.05$ \\
4 & 1.78 & 2.17 & $<0.05$ \\
Overall & 1.60 & 1.936 & $<0.05$ \\
\hline
\end{tabular}

LAA thrombus was suspected in four $(2 \%)$ patients and confirmed in two on TOE. Consent for TOE was not obtained in the other 2 patients. These data suggest that tissue harmonic imaging improves LAA visualisation on TTE. THI may increase the accuracy of TTE for the diagnosis of LAA thrombus.
Tissue harmonic imaging increases accuracy in determining left ventricular mass as measured by $\mathrm{M}$-mode echocardiography, when compared with magnetic resonance imaging

J C S Spratt, C Turnbull, D J Webb, D B Northridge; Department of Cardiology, University of Edinburgh

Background: Tissue harmonic imaging (THI) improves endocardial visualisation, we therefore hypothesised that THI would increase accuracy and decrease variability when measuring left ventricular mass (LVM) by M-mode transthoracic echo (TTE), compared with fundamental imaging and cross-referenced with the current gold standard, magnetic resonance imaging (MRI).

Methods: 20 hypertensive male patients (sitting systolic blood pressure $>150 \mathrm{~mm} \mathrm{Hg}$ ) were studied. All patients had LVM measured by both gradient-echo MRI and by TTE (ATL HDI 5000). M-mode echo measurements were taken for each patient, 2 in fundamental and 2 using THI. The order of the echo images was randomised and the images unlabelled. These images were then analysed off-line, by a blinded observer, using the ASE criteria, to calculate LVM. MRI LVM was calculated using Simpson's rule on serial short axis slices of $8 \mathrm{~mm}$ thickness.

Results: A lower intra-observer variability was observed with THI ( $4 \% v 10 \% ; \mathrm{p}, 0.01)$. There was a significant difference between THI and fundamental imaging (mean \pm SEM: $198 \pm 17 v 175 \pm 19 \mathrm{~g}$; $\mathrm{p}=$ 0.048). A lower mean difference was obtained by THI, compared to fundamental imaging, when cross-referenced with MRI (31 $\pm 8 v 42 \pm$ $11, \mathrm{p}=0.02$; difference between means $6 v 23 \mathrm{~g}$ ).

Conclusion: In hypertensive males, $\mathrm{M}$-mode echo derived from fundamental imaging has poor reproducibilty and systematically underestimates LVM compared to MRI. THI significantly improves the reproducibilty and accuracy of M-mode LVM measurement, with a greatly reduced systematic error. Widely acceptable normal ranges for LVM are not applicable when THI is used.

Longitudinal shortening velocity of the left ventricle: potential for rapid quantification of LV systolic function

D J A Price, P G Campbell, M J Stewart; South Cleveland Hospital, Middlesbrough

Background: Accurate estimation of LVEF is technically demanding and often unreliable. Visual assessment is often preferable but requires considerable experience. Longitudinal shortening of the LV, measured as mitral annular displacement (MAD) has been proposed as an alternative but can be technically challenging. We hypothesised that mitral annular displacement velocity (MADV) measured by tissue velocity imaging (TVI), which is less dependent on the acoustic window, also reflects LVEF and compared its predictive accuracy to these conventional measures.

Methods: Subjects were 48 patients with IHD; 12 female, mean age 62 years. Standard 2D echo was analysed by an experienced clinician and subjective assessment made of LV function, grading patients as requiring an ACEI or not. LVEF was measured using the method of discs, MAD measured using apical long axis $\mathrm{M}$-mode and MADV obtained from apical TVI images averaging 4 sites-septal, lateral, anterior and inferior. Ability of each measure of LV function to predict need for ACEI was compared.

Results:

\begin{tabular}{|c|c|c|c|c|c|c|}
\hline & \multicolumn{2}{|c|}{ LVEF (\%) } & \multicolumn{2}{|c|}{$M A D(\mathrm{~mm})$} & \multicolumn{2}{|c|}{$M A D V(\mathrm{~cm} / \mathrm{s})$} \\
\hline & $<40$ & $>40$ & $<9$ & $>9$ & $<5.5$ & $>5.5$ \\
\hline ACEI & 9 & 14 & 10 & 11 & 15 & 7 \\
\hline No ACEI & 0 & 26 & 3 & 23 & 7 & 19 \\
\hline
\end{tabular}

LVEF was significantly related to both MAD (Spearman's rank order correlation $r=0.43, \mathrm{p}=0.002)$ and MADV $(r=0.44, \mathrm{p}=$ 0.002 ). LVEF $<40 \%$ was $100 \%$ specific but only $39 \%$ sensitive for LV dysfunction requiring ACEI, MAD $<9 \mathrm{~mm}$ was $48 \%$ sensitive but $88 \%$ specific, MADV $<5.5 \mathrm{~cm} / \mathrm{s}$ was $73 \%$ specific and $68 \%$ sensitive. Conclusion: MADV $<5.5 \mathrm{~cm} / \mathrm{s}$ is a useful marker of LV systolic dysfunction. TVI shows promise as a practical and accurate tool in this regard. 
An echocardiographic description of the Labcor porcine replacement valve in the aortic position

J Chambers ${ }^{1}$, W Dolman', J Jawetz ${ }^{3}$, A Ramos ${ }^{4}$, J Collaco ${ }^{5}$, J Barros-Pena $^{6}$

${ }^{1}$ Guy's and St Thomas' Hospital, London; ${ }^{2}$ Sulzer Carbomedics, USA; ${ }^{3}$ Porto Alegre, ${ }^{4}$ Sao Paulo, ${ }^{5}$ Florianopolis, ${ }^{6}$ Belo Horizonte, Brazil

The Labcor valve is constructed from three separate porcine aortic cusps mounted on a scalloped, flexible stent. It has been implanted for about 10 years, and has recently been introduced into Europe. No haemodynamic data have been published. Therefore, 95 patients at four centres in Brazil were studied by one operator who was blinded to valve size. The patients were aged 58 years (SE 1.5 years; range 19-83) and $36(38 \%)$ were female. The valves were studied 34 months (SE 2.9) after implantation (range 1-120). Mean gradient was calculated using the long modified Bernoulli equation, effective orifice area (EOA) using the classical form of the continuity equation and resistance as mean gradient/flow.
There was no significant difference in peak transoartic velocity (V max) and mean gradient between the valves of different annulus size.

\begin{tabular}{lcll}
\hline & $\begin{array}{c}\text { Mean grad } \\
(\text { mm Hg) }\end{array}$ & EOA $\left(\mathrm{cm}^{2}\right)$ & $\begin{array}{l}\text { Resistance } \\
(\text { dyne.s.cm }\end{array}$ \\
\hline $21 \mathrm{~mm}(\mathrm{n}=5)$ & $13.3(1.9)$ & $1.1(0.1)$ & $457(145)$ \\
$23 \mathrm{~mm}(\mathrm{n}=31)$ & $15.3(1.2)$ & $1.4(0.1)$ & $328(40)$ \\
$25 \mathrm{~mm}(\mathrm{n}=31)$ & $13.2(1.2)$ & $1.5(0.1)$ & $242(45)$ \\
$27 \mathrm{~mm}(\mathrm{n}=13)$ & $10.6(1.3)$ & $1.8(0.1)$ & $125(25)$ \\
$29 \mathrm{~mm}(\mathrm{n}=4)$ & $9.6(2.5)$ & $1.8(0.3)$ & $100(46)$ \\
\hline
\end{tabular}

However, EOA was directly $(\mathrm{p}=0.0075)$ and resistance was indirectly related to valve size ( $\mathrm{p}=0.011$ by ANOVA). Trivial or mild regurgitation was seen in $31(38 \%)$, moderate in $3(4 \%)$ and severe in $2(2 \%)$. The regurgitation was solely paraprosthetic in $5(6 \%)$, solely through the valve in $23(28 \%)$ when it usually occurred adjacent to the commissures and in both positions in $7(9 \%)$.

Conclusion: The Labcor porcine valve has acceptable haemodynamics of forward flow. A proportion are associated with minor regurgitation and the natural history of this finding must be investigated. 